\title{
The Microstrip Antenna Design for Multiple Frequency Small Broadband FANG Mingming ${ }^{1, a}$ \\ ${ }^{1}$ Department of Information Engineering, Weihai Vocational College, Weihai 264210,China \\ afangmingming@126.com
}

Keywords: Microstrip Antenna; Multiple Frequency; Small Broadband

\begin{abstract}
Along with the miniaturization of mobile terminals, antenna miniaturization is the trend of the development, because of its small volume, light weight, with carrier conformal and easy to implement dual-band, multi-frequency work, and many other advantages. This paper designed a model with multiple inhibition zone of ultra-wideband coplanar antenna miniaturization, can restrain interference from the three wireless communication system, and has great value in industrial application. The application of the multi-frequency technology to implement system of frequency hopping and frequency sweep work mode provides a possible, to improve the anti-interference performance of the system has important significance.
\end{abstract}

\section{Introduction}

The development of wireless communication is brought more and more challenges to the design of the antenna, in the field of mobile communication and satellite communication, there is a widespread pursuit of miniaturization of multiband antenna to achieve better integration and lower cost, and with the increase of integration, as the multi-frequency antenna is difficult to meet the demand of the bandwidth of the communication terminal, the ultra-wideband antenna arises at the historic moment [1]. Microstrip antenna due to its light weight, small volume, low cost, easy and carrier conformal, easy and advantages of microwave circuit integration, can effectively meet the needs of miniaturization and more frequency ultra-wideband, thus gets more and more extensive research and application [2].

Multi-frequency technology has been successfully applied to radar, guidance, satellite communications, mobile communications and other fields, an antenna embody the function of multiple antennas, multiple frequency microstrip antenna design become another new field of antenna design. This paper first introduces the theory of microstrip antenna analysis method and the numerical analysis method, and sums up the microstrip antenna miniaturization, multiple frequencies and the bandwidth of a variety of technologies. In order to meet the needs of ultra-wideband wireless communication, this paper designed a model with multiple inhibition zone of ultra-wideband coplanar antenna miniaturization, can restrain interference from the three wireless communication system, has great value in industrial application.

\section{The analysis method of the microstrip antenna}

Using transmission line model analysis of microstrip antenna is relatively early, which is the most simple way, it is the earliest by Munson put forward in 1974. Figure 1 is its physical model [3].

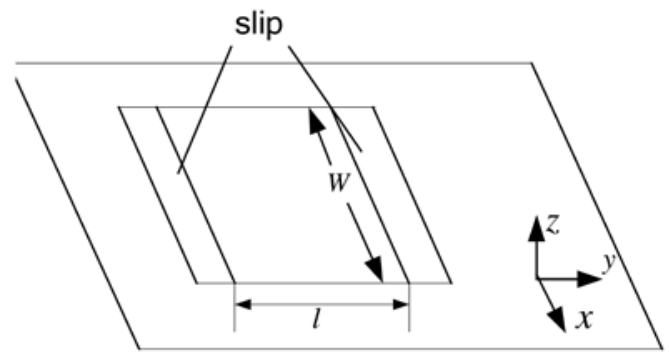

Figure 1. Physical model of transmission method 
Transmission line method is: (1) the basic hypothesis of microstrip patch and the ground plate constitute a microstrip transmission line, transmission quasi TEM wave, the wave transmission direction depends on the feed point. Segment Length $1 \approx \lambda_{\mathrm{g}} / 2, \lambda_{\mathrm{g}}$ is a quasi TEM wave wavelength.

Field in the direction of transmission is the standing wave distribution, and in the vertical direction is evenly distributed. (2) the two open end of a transmission line (beginning and end) equivalent to two radiating slot, $\mathrm{W}, \mathrm{h}$, joint aperture field is the open end field is a transmission line. At the ends of the joint plane as in microstrip patch extension. It is established according to the transmission line method of microstrip antenna equivalent circuit as shown in figure 2.

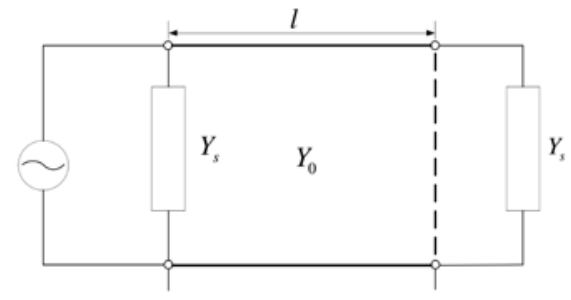

(a)

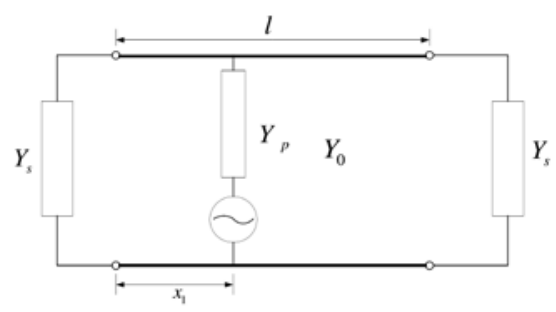

(b)

Figure 2. Equivalent circuit of transmission model for microstrip antenna

(a) microstrip-fed antenna; (b) coaxial-fed antenna

Transmission line analysis method is simple and intuitive, the advantages of small amount of calculation, but its application range is limited by a lot of, can only be used for the rectangular microstrip antenna and microstrip oscillator, although circular microstrip antenna can also have the radial transmission line and the matching, but due to the circular center feed radiation yuan radiation characteristics are not good (for the direction of the concave figure), generally will not be used [4]. For the other, such as the triangle structure of microstrip antenna, the transmission line model theory is more do not apply. In addition to the resonance point, the antenna input impedance change with frequency curve is not accurate. Susceptance part of the transmission line model is usually calculated on quasi-static method, the resulting equivalent elongation under the condition of high frequency is not accurate.

\section{Miniaturization, multiple frequencies and the bandwidth technology of microstrip antenna}

The miniaturization of microstrip antenna technology. Microstrip antenna miniaturization technology is accompanied by personal communication system and a variety of wireless communication network is appeared with the development of the mobile phone, bluetooth, and wireless local area network (LAN) of antenna miniaturization and requires a high degree of compactness. However, due to the small size of the antenna quality factor is high, cause the loss of the radiation efficiency, narrow frequency band, therefore, in the consideration of antenna miniaturization and compromising the bandwidth of the antenna gain, radiation and other technical indicators. Current, load short circuit probe or patch method is widely used in the wireless communication terminal of the current, Field map for rectangular patch can antenna is shown in figure 3-(a) be used.

The field distribution of rectangular microstrip antenna, the antenna in the two terminal (half a wavelength) resonance, is voltage antinodes points on both sides, so, in the middle of the two open terminal will there is a voltage wave node, if place a short circuit on the node voltage probe or short-circuit patch, the antenna can be a quarter of the voltage wave between nodes and antinodes resonance wavelength, so the size of the antenna is reduced to half the original, achieved the purpose of miniaturization.

The multiband microstrip antenna technology. Microstrip antenna of multi-frequency technology is mainly used in mobile phones and other wireless mobile communication terminal, realize multiband microstrip antennas work the basic pattern can be divided into two categories: 
multiple and single chip. Multi-plate method using the resonance frequency of different multiple patch work, usually smaller patch will fold on the larger patch, referred to as the product stack decay with antenna. Monolithic method is still only a patch, and the use of different modes for work at the same time, or use the resonance frequency of the load to form several different. For different shapes such as rectangular, circular, trapezoidal and pentagon patch, can put a patch is folded to another yuan for dual-band work. As shown in figure 3-(b) is a simple example.

The bandwidth of microstrip antenna technology. Impedance matching method is the most direct method to improve microstrip antenna impedance bandwidth, introduced through the antenna input ports lossless matching network, can achieve the goal of the bandwidth. In general, the impedance matching method includes coplanar and non coplanar match two forms, as shown in figure 3-(c) [5].

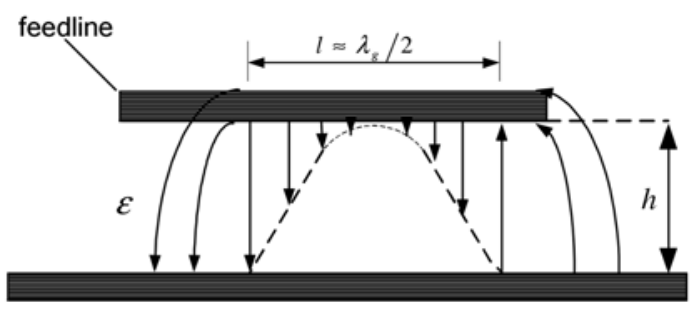

(a)

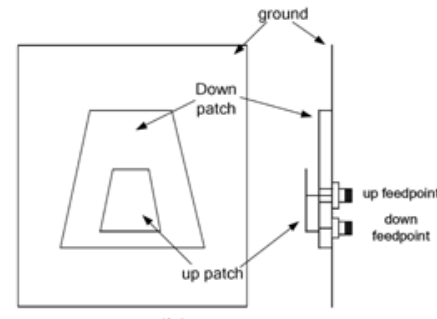

(b)

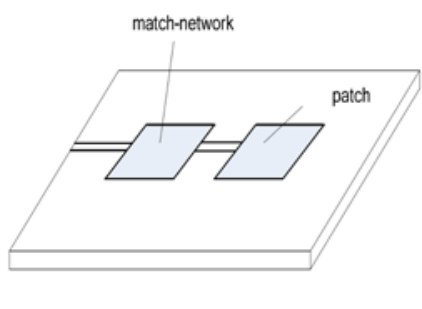

(c)

Figure 3. Miniaturization, multiple frequencies and the bandwidth technology

(a) Field map for rectangular patch antenna; (b) Structure of trapezia stacking microstrip antenna; (c) Impedance-matching method

\section{The microstrip antenna design for multiple frequency small broadband}

The multi frequency small broadband microstrip antenna structure designed is shown in figure 4, composed of two symmetrical E shape. From the structure, the double E microstrip antenna with $\mathrm{H}$ microstrip antennas are very similar, and all are symmetric structure, $\mathrm{H}$ of the antenna to be able to use analysis theory to analyze it.

As you can see by the picture, the antenna at $3.5 \mathrm{GHz}$ and $5.4 \mathrm{GHz}$ with two narrow band, WiMAX and WLAN communication standard. Between $3.5 \mathrm{GHz}$ to $5.4 \mathrm{GHz}$, however, the loss is bigger, lead to poor antenna out-of-band flatness, this is because in the $3.5 \mathrm{GHz}$ and $5.4 \mathrm{GHz}$ between a resonance point, and the feeding way and not completely dropped the inhibition of this model. In fact, this type of antenna has at least four resonant frequency, only through the feed point position changes will not need frequency suppression will not remove, therefore, when using symmetric structure design multiband antenna, the resonant frequency, must choose carefully use as far as possible to avoid unable to inhibit out-of-band resonance point.
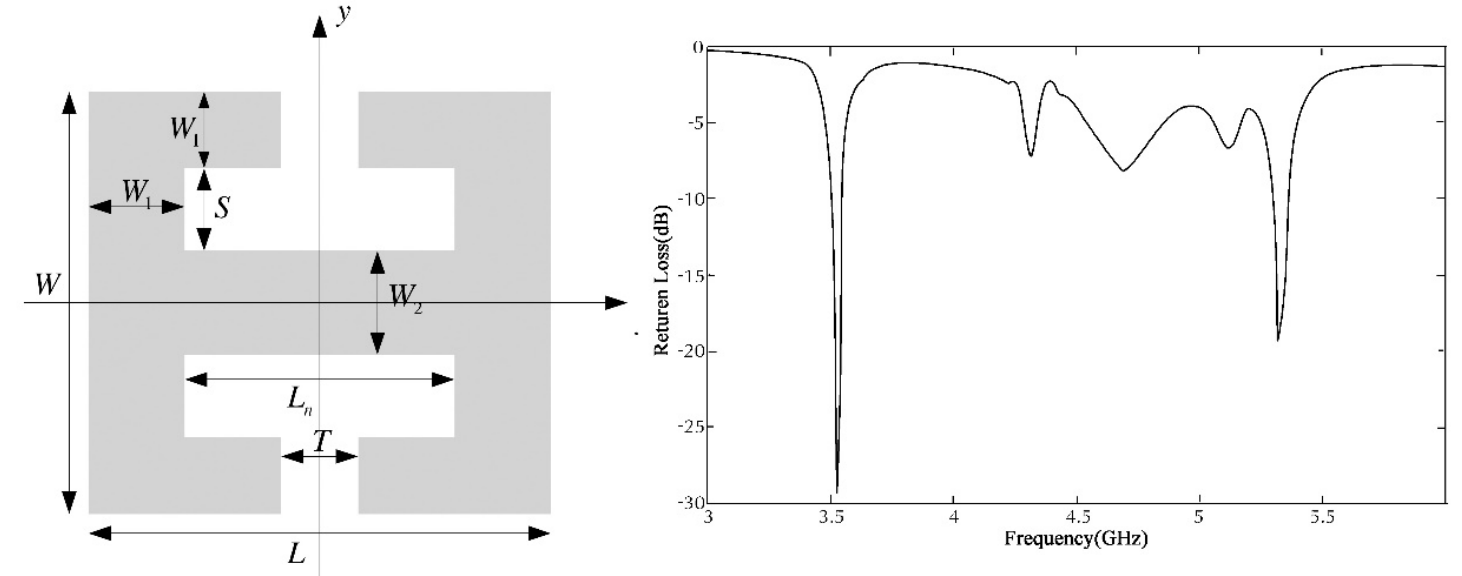

Figure 4. The structure of multi frequency small broadband microstrip antenna 


\section{Conclusion}

Microstrip antenna with small volume, light weight, thin section, easy to shape and easy processing with aircraft, easy and active devices and circuit integrated into a single module, and many other advantages, has been widely researched and applicated. This paper fully studies the existing microstrip antenna miniaturization and multiple spectrum technology, the comprehensive existing common under the premise of microstrip antenna loading technology, realize the miniaturization of a novel multiband microstrip antenna design ideas and research methods, and on this basis, we design a hybrid loading realize the miniaturization of multiband microstrip antenna, has carried on the thorough analysis to the performance of the antenna, the simulation optimization and test the paper puts forward the design ideas and research methods of analysis and verification of the complete.

\section{Reference}

[1] B. Zhou, H. Li, and X. Zou: Progress In Electromagnetics Research, Vol.120 (2011), p.235.

[2] M.C. Tang, S. Xiao, and T. Deng: Antennas and Propagation, Vol.59 (2011) No.4, p.1372

[3] M.R. Chaharmir, J. Shaker, and N. Gagnon: Antennas and Propagation, Vol.58 (2010) No.9, p.2875

[4] J. Anguera, A. Andujar, and C. Garcia: Antennas and Propagation, Vol.38 (2008) No.12, p.1025 2013, 61(7): 3782-3789.

[5] F. Viani, M. Salucci, F. Robol : Journal of Electromagnetic Waves and Applications, Vol.26 (2012) No.4 p.480 